\title{
Editorial
}

\section{Proceedings of the Nutrition Society: 2000 and beyond}

It seems fitting that the Proceedings of the Nutrition Society should continue to change and develop as the Nutrition Society as a whole changes and develops; the emphasis being on increased accessibility to the Society's affairs and journals brought about by 'electronification', including the inclusion of the Proceedings in several on-line indexing services. A huge amount of effort has gone into producing the Nutsoc website, and updating it regularly, as well as putting in the links that allow some subscribers to read the journals on-line. The Society and our publishers, CABI Publishing, are currently investigating the possibility of letting members subscribe to their choice of electronic or paper versions of the four journals, perhaps at special rates. The New Year will see the inclusion of the Proceedings, alongside the Society's other journals, on the ingenta Journals service at http://www.ingenta.com.

Another aspect of the improvements made possible by using technology relates to the handling of the Original Communications; these will now be submitted, put onto the website, and edited and printed from disk, which should (after the inevitable teething troubles!) make the whole process easier for everyone (see website/Gazette for new 'Directions' etc.). This is not being undertaken lightly, but after much planning and consideration of all of the things that might go wrong...go wrong...go wrong... vol. 59 of the Proceedings will be published in six issues (rather than four) this year, two of which will contain only Original Communications (OC), while the symposium papers will go into the other four. Thus, the first issue to appear will contain all the OC from the Summer meeting (July 1999), Irish Section annual meeting, and joint meeting with the Spanish Nutrition Society (SEN) in Pamplona (September 1999), which will be available sooner than if they had waited to be published with the symposium papers. The second OC issue will contain the first of the OC to be handled electronically as outlined previously, which will be an interesting exercise! This increased speed of publication will benefit authors, and will also allow separate marketing by CABI Publishing of the symposium and $\mathrm{OC}$ issues.

The symposia being published this year include those presented at the Summer meeting, which had the overall theme of 'Nutrition of the young'. The roles of the nutrients in healthy babies were considered in detail, including the part played by micronutrients such as retinoic acid, I, polyamines and folic acid as modulators of development. Sick children, their nutritional requirements and the effects of perturbations in the supply of nutrients were also covered, including new research on adequacy of breast-feeding. This theme was continued in the symposium on the influence of maternal reserves and early lactation on the development of young animals. The Irish Section symposium took the older child as its topic 'Growing up with good nutrition: a focus on the first two decades', with papers on food allergy, poverty, eating and exercise patterns, prevention of osteoporosis, secular trends in growth, body image concerns, and new cellular and genetic insights into the acquisition of obesity.

The Pamplona meeting was concerned entirely with obesity, considering the fundamental causes of the condition, both environmental and physiological (yes, leptin did feature here!), integrated with a fascinating and revealing trio of papers on neuroendocrine activity controlling food intake and body weight. Obesity was also a consideration at a joint meeting on 'Reducing cardiovascular disease risk: today's achievements - tomorrow's opportunities' from which will be published short summaries of the papers presented: a comprehensive collection covering everything from the investigation of genetic determinants of blood lipids and drug responsiveness, to current and future treatments of risk factors by diet, drugs and exercise; with a real attempt being made to assess the actual benefits that might be gained by measures designed to modify risk while acknowledging the practical difficulties involved.

The joint meeting with BAPEN has generated some papers of interest to those looking after severely-injured patients, considering both the effects of the illness on nutritional status, and the means by which nutrition can be used to give the best possible help in management and recovery. Part of this picture involves the possible modulation of the immune system by nutritional means, a concept of increasing interest as clinicians, immunologists and nutrition scientists begin to comprehend the mechanisms that, once investigated and understood, could potentially be manipulated for the benefit of patients. This area will be investigated in detail by speakers at the Winter meeting on 'Nutrition and immunity', which will, I hope, produce papers giving valuable insight into this complex field for those of us who are not familiar with it.

K. M. Younger

Editor

Proceedings of the Nutrition Society 